\title{
BMJ Open IL-23 blockade with guselkumab potentially modifies psoriasis pathogenesis: rationale and study protocol of a phase $3 b$, randomised, double-blind, multicentre study in participants with moderate-to-severe plaque-type psoriasis (GUIDE)
}

\author{
Kilian Eyerich, ${ }^{1}$ Peter Weisenseel, ${ }^{2}$ Andreas Pinter, ${ }^{3}$ Knut Schäkel, ${ }^{4}$ \\ Khusru Asadullah, ${ }^{5}$ Sven Wegner, ${ }^{6}$ Ernesto J Muñoz-Elias, ${ }^{7}$ Holger Bartz, ${ }^{8}$ \\ Friedmann $\mathrm{J} \mathrm{H}$ Taut, ${ }^{9}$ Kristian Reich (D) ${ }^{10}$
}

To cite: Eyerich $\mathrm{K}$, Weisenseel $\mathrm{P}$, Pinter A, et al. IL-23 blockade with guselkumab potentially modifies psoriasis pathogenesis: rationale and study protocol of a phase $3 b$, randomised, double-blind, multicentre study in participants with moderate-to-severe plaque-type psoriasis (GUIDE). BMJ Open 2021;11:e049822. doi:10.1136/ bmjopen-2021-049822

- Prepublication history for this paper is available online. To view these files, please visit the journal online (http://dx.doi org/10.1136/bmjopen-2021 049822).

Received 09 February 2021 Accepted 03 August 2021

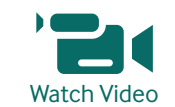

bmjopen.bmj.com

Check for updates

(C) Author(s) (or their employer(s)) 2021. Re-use permitted under CC BY-NC. No commercial re-use. See rights and permissions. Published by BMJ.

For numbered affiliations see end of article.

Correspondence to

Dr Kristian Reich;

kreich@jerucon.com

\section{ABSTRACT}

Background Guselkumab is an interleukin (IL)-23 pathway blocker with proven efficacy in patients with moderate-to-severe plaque psoriasis. Early intervention with guselkumab may result in changes to the clinical disease course versus later intervention.

Methods and analysis Here we present the rationale and design of a phase $3 b$, randomised, double-blind, multicentre study (GUIDE), comparing treatment effects of guselkumab in patients with short ( $\leq 2$ years) or longer ( $>2$ years) duration of plaque-type psoriasis, measured from first appearance of psoriatic plaques. Participants achieving skin clearance (Psoriasis Area and Severity Index $(\mathrm{PASI})=0$ ) by week 20 and maintaining complete clearance at week 28 visit ('super-responders' (SRe)) will be randomised to continue approved maintenance dosing every 8 weeks ( $q 8 w$ ) versus an investigational maintenance dosing interval of 16 weeks (q16w) until week 68. Primary endpoint: proportion of participants in the q8w vs q16w arms with absolute PASI $<3$ at week 68. Participants with PASI $<3$ at week 68 will be withdrawn from guselkumab treatment for up to 48 weeks. Participants not achieving SRe criteria (non-SRe) will remain in the study with q8w guselkumab dosing through week 68. Additional to serum samples obtained from all patients, skin biopsies and whole-blood samples will be taken from SRe and non-SRe participants at various time points in optional substudies. Analyses include: genetics; immunophenotyping (fluorescence-activated cell sorting); gene and protein expression profiling; immunohistology. By merging clinical endpoints with mechanistic findings, this study aims to elucidate how IL-23 blockade with guselkumab can modify the disease course by altering molecular and cellular drivers that cause relapse after treatment withdrawal, particularly among SRe.

Ethics and dissemination Approval obtained from ethics committee Medical Council Hamburg, Germany (PVN5925). GUIDE is compliant with the Declaration of Helsinki.

\section{Strengths and limitations of this study}

$\Rightarrow$ GUIDE is a phase $3 \mathrm{~b}$, randomised, double-blind, parallel-group, multicentre, multinational study addressing new therapeutic strategies in the treatment of high-burden psoriasis.

$\Rightarrow$ Clinical data combined with state-of-the-art immunological and molecular analyses using blood and skin tissue obtained from participants will assess the underlying immunopathogenesis and chronic nature of psoriasis.

$\Rightarrow$ If successful, the study may establish the first approach for identifying endotypes of psoriasis where early intervention may influence the long-term course of disease.

$\Rightarrow$ Mechanistic substudies are exploratory in nature and will be correlated to clinical observations.

$\Rightarrow$ Translation from a controlled trial to a real-world setting is limited.

Trial registration number Registered at ClinicalTrials.gov (NCT03818035). All primary endpoint results (prespecified analyses) will be submitted to peer-reviewed, international journals within 18 months after primary completion date.

\section{INTRODUCTION}

Plaque psoriasis is a common, chronic immunemediated inflammatory disease characterised by plaques of red, dry, itchy and scaly skin that can manifest in all skin areas and vary in size from a few millimetres in diameter to covering large parts of the body surface. Psoriasis is associated with multiple comorbidities including cardiovascular disease, hypertension, metabolic syndrome, chronic kidney disease and 
psoriatic arthritis. ${ }^{1}$ Thus, plaque psoriasis patients carry a high burden of disease that extends beyond the visible signs in the skin. Peak disease onset is in early adulthood and the disease course is chronic; therefore, the need for treatment is lifelong. ${ }^{2-4}$ Currently, patients with moderate-tosevere plaque psoriasis are usually treated for several years with a combination of topicals, UV-light or conventional systemic immunosuppressive drugs. Commonly, patients switch to biological therapy several years after diagnosis, and most often when a satisfactory response has not been achieved, contraindications exist, or an adverse event forces a switch. ${ }^{5-7}$

The immunopathogenesis of psoriasis is based on a complex interplay between genetic susceptibility, environmental triggers and components of innate and adaptive immunity. At its core, psoriasis is a T-cell-mediated disease, in which dysregulation of the immune system in the skin promotes inflammatory responses and results in abnormal proliferation of keratinocytes and extensive infiltration of inflammatory cells. ${ }^{89}$ With the recognition that the interleukin (IL)-23/IL-17A/F immune axis is central to the pathogenesis of psoriasis, and that therapeutics targeting IL-23, IL-17A, IL-17A/F represent the most advanced and effective treatment options for patients, IL-23 has emerged as a 'master regulator' in psoriasis. ${ }^{2}$ IL-23 promotes terminal differentiation, expansion and maintenance of IL-17 producing cells (T17), expressing $\mathrm{CD}^{+}$(T helper 17 (Th17)) or $\mathrm{CD}^{+}$(Tc17) T cells. ${ }^{5-79-11}$ IL-23 has also been reported to impair the function of regulatory $\mathrm{T}$ cells $\left(\mathrm{T}_{\text {reg }}\right)$ and to promote the differentiation of $\mathrm{T}_{\text {reg }}$ into Th17-like cells in psoriatic patients, thereby dampening anti-inflammatory $\mathrm{T}_{\text {reg }}$ responses. ${ }^{12-15}$ Further, IL-23 appears to be involved in the differentiation and survival of pathogenic tissue-resident memory $\mathrm{T}$ cells $\left(\mathrm{T}_{\mathrm{RM}}\right)$, ${ }^{16}$ which are thought to be responsible for recurrence of psoriatic skin lesions in previously affected sites. ${ }^{17}$ The physiological role of IL-23 in the human immune system is not well established. Observations of reduced antigen-specific immunoglobulins of all isotypes and a diminished delayed type hypersensitivity in IL-23p19-deficient mice point towards a defect at the level of $\mathrm{T}$ cells. However, the safety profile of guselkumab in clinical trials in psoriasis patients is not consistent with a generalised memory $\mathrm{T}$ cell defect. ${ }^{18-20}$

By targeting pathogenic $\mathrm{T}$ cells, in particular $\mathrm{T}_{\mathrm{RM}}$, in affected tissue, inhibition of IL-23 may have a profound effect on the pathophysiology of psoriasis that might extend beyond clearance of psoriasis plaques while on treatment. Guselkumab, an antibody that binds to the p19 subunit of IL-23, was the first approved selective inhibitor of IL-23 for the treatment of chronic plaque psoriasis.

Here, we discuss the concept of modifying the disease course towards long-term remission as a potential novel treatment goal for psoriasis. Also, we discuss the recently commenced Phase 3b, Randomised, Double-blind, Active-controlled, Multicentre Study to Evaluate Further Therapeutic Strategies with Guselkumab in Subjects with Moderate-to-Severe Plaque Psoriasis (GUIDE) study, which seeks to test the hypothesis that in psoriasis, early intervention with an IL-23 inhibitor could lead to better clinical responses and more durable maintenance of response after drug withdrawal.

\section{The pathogenesis of psoriasis}

IL-23 is a 'master regulator' cytokine in psoriasis

IL-23 has emerged as a 'master regulator' cytokine in many chronic inflammatory diseases, particularly in psoriasis. $^{2} 3$ 8-11 21-24 IL-23 bridges the innate and adaptive immune systems, as it acts on T cells as well as innate immune cells (eg, natural killer cells, macrophages, dendritic cells and innate lymphoid cells). IL-23 normally confers immunity against bacterial and fungal infections. However, dysregulated production of IL-23 promotes the development of chronic inflammation. It signals through the IL-23 receptor, which is believed to be expressed on several types of immune cells, including $\mathrm{T}$ cells, natural killer cells, neutrophils, mast cells, innate lymphoid cells and macrophages. ${ }^{25-27}$

Binding of IL-23 to its receptor primarily activates signal transducer and activator of transcription (STAT3), which has critical functions in Th17 and Tc17 cell activation, differentiation, proliferation and survival. STAT3 directly regulates the genes encoding IL-17A, IL-17F and IL-23R ${ }^{28}$ and indirectly controls expression of the retinoic acid receptor-related orphan receptor (ROR) $\gamma t$ transcription factor, a regulator of Th17 differentiation. ${ }^{29}$

\section{Skin resident memory T cells provide rapid local immune responses}

Tissue-resident memory $\mathrm{T}$ cells are a recently identified subset of non-circulating memory $\mathrm{T}$ cells that persist longterm in peripheral tissues. In both mice and humans, these cells express CD69, and subsets also express CD103 and CD49a. ${ }^{1730} \mathrm{CD} 69$ appears to be involved in retention of $T_{R M}$ in peripheral tissues. CD103 is a known ligand of E-cadherin, a homotypic adhesion molecule expressed by epithelial cells in barrier tissues. ${ }^{31}$ Under healthy conditions, $\mathrm{T}_{\mathrm{RM}}$ differentiate and accumulate in tissues following infections or vaccinations to provide rapid local immune responses on re-exposure to pathogens. However, $\mathrm{T}_{\mathrm{RM}}$ may also develop after sensitisation to selfantigens, and thus be involved in the pathogenesis of autoimmune disorders such as psoriasis. ${ }^{1731-34}$

One of the main challenges in the treatment of psoriasis is recurrence of psoriatic skin lesions, usually at the same locations on the body. The persistent localised presence of pathogenic $T_{R M}$ derived from clonally expanded autoreactive T cells may explain this phenomenon. ${ }^{172-34}$ The first hint for the involvement of $\mathrm{T}_{\mathrm{RM}}$ in psoriasis was provided by the unexpected observation that blockade of E-selectin, which inhibits $\mathrm{T}$ cell migration from the blood into the skin, was ineffective in the treatment of psoriasis. ${ }^{35}$ Skin transplant experiments in mice demonstrated that non-lesional, prepsoriatic human skin grafts developed into psoriasis lesions after transplantation onto immunodeficient mice. ${ }^{36} 37$ These lesions emerged 
through activation and proliferation of resident $\mathrm{T}$ cells transferred with non-lesional skin grafts.

Transcriptomic analysis of skin biopsies from human patients revealed that a subset of disease-related genes remained abnormally expressed in healed lesional skin responding to antitumour necrosis factor $\alpha$ treatment. ${ }^{38}$ These included $\mathrm{T}$ cell-associated genes, as well as genes associated with 'structural' cell types, such as keratinocytes, which collectively represent a 'molecular scar' in resolved psoriatic lesions (figure 1A). Additional histological studies verified the presence of residual dermal $\mathrm{CD}^{+} \mathrm{T}$ cells in resolved psoriatic skin. IL-17A ${ }^{+} \mathrm{CD} 8^{+} \mathrm{T}_{\mathrm{RM}}$ and $\mathrm{IL}-22^{+} \mathrm{CD} 4^{+} \mathrm{T}_{\mathrm{RM}}$ cells were found to persist in the epidermis, possibly representing a form of disease memory in clinically healed regions of psoriasis. ${ }^{16} 3940$ In particular, a distinct population of epidermal $\mathrm{CD}^{+} \mathrm{T}$ cells coexpressing CD103, CCR6 and IL-23R was highly enriched in resolved lesions. ${ }^{16}$ These findings point to a possible scenario, in which $\mathrm{CD}^{+} \mathrm{T}_{\mathrm{RM}}$ drive inflammation and recruitment of circulating leucocytes into the tissue through IL-23-dependent IL-17A production, while $\mathrm{CD} 4^{+}$ $\mathrm{T}_{\mathrm{RM}}$ promote keratinocyte activation and development of acanthosis through production of IL-22. CD49a was recently identified as a phenotypic marker for the differentiation of $\mathrm{CD}^{+} \mathrm{CD} 103^{+} \mathrm{T}_{\mathrm{RM}}$ on a functional basis. ${ }^{30} \mathrm{CD} 49 \mathrm{a}^{-} \mathrm{T}_{\mathrm{RM}}$ produced IL-17, preferentially expressed IL-23R, and were enriched in psoriatic lesions, whereas $\mathrm{CD}_{4} \mathrm{a}^{+} \mathrm{T}_{\mathrm{RM}}$ produced interferon- $\gamma$ and were enriched in the skin of vitiligo patients. Another recent study detected IL-17-producing $\alpha \beta$ T cell clones with psoriasis-specific antigen receptors in residual $\mathrm{T}$ cell populations of clinically resolved psoriatic skin. ${ }^{41}$ These cells were postulated to represent lesion-initiating pathogenic T cells in psoriasis. ${ }^{41}$ Increasing evidence indicates that the majority of $\mathrm{CD}^{+} \mathrm{T}$ cells in psoriasis plaques are present as $\mathrm{T}_{\mathrm{RM}}{ }^{42}$ implying a predominant role for $\mathrm{CD}^{+} \mathrm{T}_{\mathrm{RM}}$ in psoriasis recurrence. In biopsies obtained from psoriatic lesions, the majority of IL-17A-producing $\mathrm{CD} 8^{+} \mathrm{T}$ cells were $\mathrm{T}_{\mathrm{RM}}$, whereas the majority of IL-17A-producing $\mathrm{CD}^{+} \mathrm{T}$ cells were non- $\mathrm{T}_{\mathrm{RM}}$ (defined as $\mathrm{CD}^{+} / \mathrm{CD}^{+} / \mathrm{CD} 103^{-} / \mathrm{CD} 49^{-} \mathrm{T}$ cells). ${ }^{43}$

\section{$\mathrm{T}_{\text {reg }}$ display functional deficits in psoriasis}

$\mathrm{T}_{\text {reg }}$, which account for $5 \%-10 \%$ of skin resident $\mathrm{T}$ cells, sustain immune homeostasis and maintenance of selftolerance by suppressing inflammation, and specifically effector $\mathrm{T}$ cells. ${ }^{44} \mathrm{~T}_{\text {reg }}$ are characterised by high expression of Foxp3, a key transcription factor essential for their function, and IL-25, the IL-2 receptor alpha chain. $\mathrm{T}_{\text {reg }}$ proliferate under conditions similar to those found in inflamed skin. ${ }^{12} 334546 \mathrm{~T}_{\text {reg }}$ and T17 cells share naïve T cells as their common precursor, and their differentiation programmes are reciprocally interconnected (figure $1 \mathrm{~B}$ ). $\mathrm{T}_{\text {reg }}$ exert anti-inflammatory effects by suppressing the activity of other skin-resident $\mathrm{T}$ cells, such as $\mathrm{T} 17$ cells. ${ }^{33}$ The balance between proinflammatory T17 cells and immunosuppressive $T_{\text {reg }}$ plays a critical role in the pathogenesis of several autoimmune diseases. ${ }^{9} 4547$ Studies finding elevated T17/ $\mathrm{T}_{\text {reg }}$ ratios in patients with rheumatoid arthritis (RA), psoriasis, multiple sclerosis, non-alcoholic fatty liver disease and inflammatory bowel disease support this notion. ${ }^{45} 48$ In psoriasis, dysfunction of $\mathrm{T}_{\text {reg }}$ has been suggested as a potential mechanism behind the imbalance of $\mathrm{T}_{\text {reg }}$ and pathogenic T cells. ${ }^{1499}{ }^{50}$ Psoriatic $\mathrm{T}_{\text {reg }}$ demonstrate proliferative and functional deficits that may permit hyperproliferation of pathogenic T cells. ${ }^{50}$ Recently, STAT3-phosphorylation induced by IL-23, IL-6 and IL-21 was revealed to impair $\mathrm{T}_{\text {reg }}$ function. ${ }^{15}$ Lineage plasticity and interconversion between $\mathrm{T}_{\text {reg }}$ and $\mathrm{T} 17$ cells could represent another mechanism of $\mathrm{T}_{\text {reg }}$ dysfunction. ${ }^{3}{ }^{45}$ Since Foxp3 and RORc are lineagedetermining transcription factors for $\mathrm{T}_{\text {reg }}$ and $\mathrm{T} 17$ cells, respectively, their balance regulates the fate of $\mathrm{T}_{\text {reg }}$, thereby affecting the $\mathrm{T} 17 / \mathrm{T}_{\text {reg }}$ ratio. ${ }^{45}$ For example, instability of Foxp3 in $\mathrm{T}_{\text {reg }}$ was reported to lead to the generation of potentially autoreactive effector T cells. ${ }^{46}$ Another pathogenic mechanism may be the phenotypic conversion of $\mathrm{T}_{\text {reg }}$ into Th17 cells. ${ }^{14} 49$ Peripheral blood derived $\mathrm{T}_{\text {reg }}$ from patients with severe psoriasis have an enhanced propensity to convert into IL-17A expressing cells, which is associated with progressive loss of Foxp3 and increased expression of ROR $\gamma$ t. This shift from $\mathrm{T}_{\text {reg }}$ to a Th17-related phenotype is promoted by IL-23. ${ }^{12}$ The finding that IL- $17 \mathrm{~A}^{+} \mathrm{Foxp} 3^{+} \mathrm{CD} 4^{+}$ positive cells are present in psoriasis plaques suggests that conversion of $\mathrm{T}_{\text {reg }}$ into IL-17A-producing cells may also occur in vivo. ${ }^{12}{ }^{\text {reg }}$ more recent study confirmed that IL-23 drives plasticity of $\mathrm{T}_{\text {reg }}$ by inducing Foxp $3^{+} \mathrm{ROR} \gamma \mathrm{t}^{+} \mathrm{IL}-17 \mathrm{~A}$ cells, and that this process is regulated by ROR $\gamma t$ signalling. ${ }^{13}$

\section{Expanding the concept of modifying disease memory in psoriasis}

Overall, current knowledge suggests that epidermal $\mathrm{T}_{\mathrm{RM}}$ generated by immune challenges in peripheral tissues persist in previously lesional psoriatic skin after healing. In response to triggers that may induce recurrence of disease, $\mathrm{T}_{\mathrm{RM}}$ can mount rapid, localised immune responses featuring the production of cytokines with critical roles in the pathogenesis of psoriasis. Recent findings suggest that IL-23 is involved in the differentiation and survival of pathogenic $\mathrm{T}_{\mathrm{RM}}$. Given that investigations of the pathogenic role of $T_{R M}$ in psoriasis are still at an early stage, many questions still need to be addressed in this field. This study will explore the question, if the reduction or even eradication of $\mathrm{T}_{\mathrm{RM}}$ through IL-23 inhibition by a monoclonal antibody such as guselkumab can be achieved and leads to improvement of clinical disease beyond the period of treatment. Explorative analysis of this study will link underlying cellular changes (ie, the frequencies of $\mathrm{T}_{\mathrm{RM}}$ and $\mathrm{T}_{\mathrm{reg}}$ ) to the clinical course of disease recurrence during the withdrawal period (week 68-week 116). By comparing subjects starting the drug withdrawal period with complete skin clearance (absolute Psoriasis Area and Severity Index (PASI) score of 0 ) with those with some remaining plaques (absolute PASI score $>0$ and $<3$ ) we aim to gain insights into the correlation between speed of disease recurrence and changes of $\mathrm{T}$ cell frequencies. Biopsies will therefore be taken at week 68 and at the timepoint of loss of disease control (PASI $>5$ ). This immunological understanding of long-term drug-free remission would be a step towards 

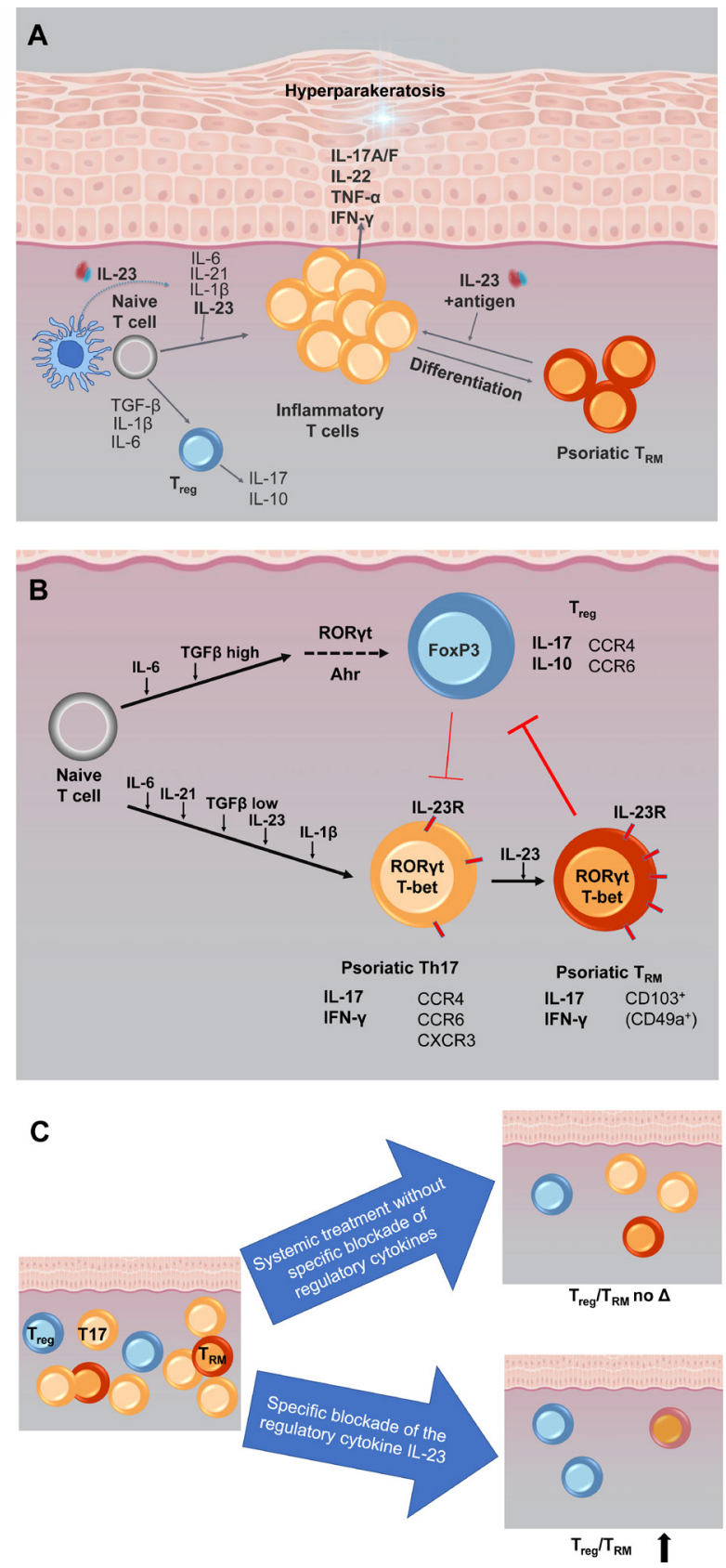

Figure 1 Current pathogenic model of psoriasis. ${ }^{212} 144165$ (A) T cell subsets in psoriasis, their differentiation, function and phenotype. High TGF- $\beta$ concentration promotes IL-10 and IL-17 producing $T_{\text {reg }}$ differentiation, while the presence of IL-6, TGF- $\beta$, IL-1- $\beta$ and IL-21 promote dominant Th17 cell differentiation expressing an IL-17/IFN- $\gamma$ cytokine signature. (B) In early psoriasis, environmental stimuli in combination with a loss of tolerance activate the innate immune system, leading to the production of IL-23 by dermal DCs and macrophages. IL-23 then drives activation and expansion of T17 cells which subsequently generate a cytokine milieu that promotes a feedforward inflammatory cascade in epidermal keratinocytes, leading to parakeratosis and psoriatic lesions. IL-17 autoamplifies its signal by triggering the production of chemokines by activated keratinocytes, which subsequently recruits more T17 cells and other immune cells (eg, neutrophils, DCs and macrophages). Persistent high levels of IL-23 in psoriatic skin sustain IL-17 production, thus fuelling the self-amplifying inflammatory process. As psoriasis progresses to a more chronic state, sustained high levels of IL-23 in combination with low concentrations of TGF- $\beta$ promotes IL-23R expression, favouring $T 17$ cell differentiation and supressing $T_{\text {reg }}$ differentiation. $T_{R M}$ are a subset of non-circulating memory $\mathrm{T}$ cells that persist long-term in peripheral tissues and are characterised by the markers CD69 and CD103. (C) Inhibition of the regulatory cytokine IL-23 is hypothesised to lead to long-lasting therapeutic effects by restoring a 'physiological' $T_{\text {reg }} / T_{R M}$ balance. This is in contrast to the blockade of an effector cytokine, which leads to reduction of inflammatory cells but has less effect on the relative numbers of proinflammatory and anti-inflammatory T cells. Ahr, aryl hydrocarbon receptor; CCR, chemokine receptor; CD, cluster of differentiation; CXCR, CXC chemokine receptor; DC, dendritic cell; IFN, interferon; IL, interleukin; RORyt, retinoic acid receptor-related orphan receptor transcription factor; T17, IL-17 producing T cells; T-bet, T box protein expressed in T cells; TGF, transforming growth factor; Th17, T helper $17 ; \mathrm{T}_{\text {reg, }}$, regulatory $\mathrm{T}$ cell; $\mathrm{T}_{\mathrm{RM}}$, tissue-resident memory $\mathrm{T}$ cell. 
disease modification, a principle that has been described for treatment of other autoimmune diseases (eg, RA and Crohn's disease) with biological agents, ${ }^{51}{ }^{52}$ but this is not yet clearly defined for psoriasis.

\section{Early intervention with IL-23 inhibitors may lead to long- lasting and disease-modifying effects}

Psoriasis predominantly presents as a skin disease and affects patients' quality of life physically, emotionally and socially. Treatment should aim to improve overall prognosis by reducing the severity of disease and preventing relapse, with the ultimate goal of modifying the course of disease. However, it is well known that initial approaches to treatment of even moderate-to-severe psoriasis are based on topical therapy, which is typically not highly effective. ${ }^{53}$ The current German guideline ${ }^{54}$ proposes the use of biologics when failure of conventional firstline therapeutics in plaque psoriasis can be anticipated. Thus, therapy with biologics is often not started until other systemic options have failed, which could be several years after the onset of disease. According to the Dutch registry BioCAPTURE, between 2005 and 2015 the median disease duration at the start of first-line conventional systemic therapy in patients with severe psoriasis was 11.0 years and the median disease duration at start of treatment with biologics was 18.9 years. ${ }^{55}$

In other immune-mediated inflammatory diseases such as RA, early intervention with biologics has been shown to increase remission rates, improve symptoms and halt joint damage. In addition, patients with RA with recent onset disease $(<2$ years) exhibited better treatment responses than those with long-standing disease.$^{53}$ It can be hypothesised that, in the treatment of psoriasis, a 'hit hard and early' approach might also result in high response rates with long-lasting remission after treatment stop, provided that the 'hard hit' aims at the right target. In particular, early intervention with selective and direct IL-23 inhibitors which specifically interfere with the major disease-driving pathway in psoriasis could prove beneficial. Early intervention with IL-23 inhibitors may have the potential of restoring a 'healthy' $\mathrm{Th} 17 / \mathrm{T}_{\text {reg }}$ balance and controlling $\mathrm{T}_{\mathrm{RM}}$ levels, thus promoting a long-lasting and possibly disease-modifying therapeutic effect (figure 1C).

In clinical studies, direct IL-23 inhibition has been shown to successfully induce remission in psoriasis with clearance rates that exceed those achieved by other nonbiological and biological systemic psoriasis treatments. Approximately $44 \%$ of patients achieved complete clearance (PASI score $=0$ ) and about $70 \%$ of patients achieved nearly complete clearance (PASI score $\leq 1$ ) after 24 weeks of treatment with guselkumab. ${ }^{56-58}$ Recently presented long-term data for guselkumab demonstrated that efficacy for treatment of moderate-to-severe psoriasis was maintained through 5 years. ${ }^{59}$ Interestingly, some patients, who had achieved a 90\% improvement from their baseline PASI score (PASI 90 response) with guselkumab, sustained their clinical response for several months after withdrawal of treatment, after complete washout of the drug (five half-lives; mean $\mathrm{T}_{1 / 2}: 15-18$ days). ${ }^{58}$ Maintenance of PASI 90 response after drug withdrawal was associated with continued suppression of IL-17A, IL-17F and IL-22. ${ }^{60-62}$ In exploratory analyses, ${ }^{63}$ participants with shorter disease duration ( $\leq 2$ years) seemed to have a higher maintenance of response rate after drug withdrawal than participants with a longer history of disease (ie , >10 years). Furthermore, achieving an absolute PASI score of 0 at week 28 was associated with long-term maintenance of PASI 90 response: long-term maintenance of PASI 90 response was achieved by $45.8 \%$ of participants who achieved an absolute PASI score of 0 at week 28 compared with $24 \%$ of participants who did not (OR of 2.66; $\mathrm{p}<0.005$, Fisher's exact test). These results imply that a distinct subset of patients with short disease duration ( $\leq 2$ years) and striking clinical response to guselkumab treatment (absolute PASI score of 0 at week 28) may more readily achieve long-term, drug-free disease control. Overall, preliminary clinical data suggest that early intervention with selective IL-23 inhibitors may have the potential to wield a profound effect in psoriasis that extends beyond short-term clearance of psoriatic skin lesions, and could instead lead to a long-term, sustained clinical response in certain patients.

\section{MATERIALS AND METHODS}

\section{Evaluating disease modification in clinical trials: the GUIDE} study

Currently, therapeutic intervention is individualised depending on the patient's measurable severity of disease, which is traditionally assessed by the PASI and Investigator Global Assessment tools. Since it is difficult to predict which biological treatment may induce an adequate clinical responses in a given patient, the choice of an optimal therapeutic agent remains a challenge, and therapies are often characterised by cycles of medication trial and error. $^{2}$ Biomarker-based assessment of clinical response to treatment and corresponding precision dosing may become an attractive approach towards a more individually tailored therapy in psoriasis.

GUIDE (NCT03818035) is a phase 3b randomised, double-blind, parallel-group, multicentre trial, including $>850$ adult participants with moderate-to-severe plaque type psoriasis, of whom approximately $40 \%$ will have disease duration of $\leq 2$ years. In part 1 of the study, all participants receive guselkumab at week 0 (baseline), and weeks 4, 12, 20 and 28 (figure 2) following the standard guselkumab treatment regimen. It is assumed that about 280 participants will qualify to be categorised as superresponders (SRe), defined as those with an absolute PASI score of 0 at both week 20 and week 28 . SRe are then randomly assigned to either of two treatment groups: Group 2a receives guselkumab $100 \mathrm{mg}$ by subcutaneous injection every 8 weeks $(\mathrm{q} 8 \mathrm{w})$ and group $2 \mathrm{~b}$ receives the same dose every 16 weeks (q16w). In addition, participants with disease duration $\leq 2$ years are equally distributed to 


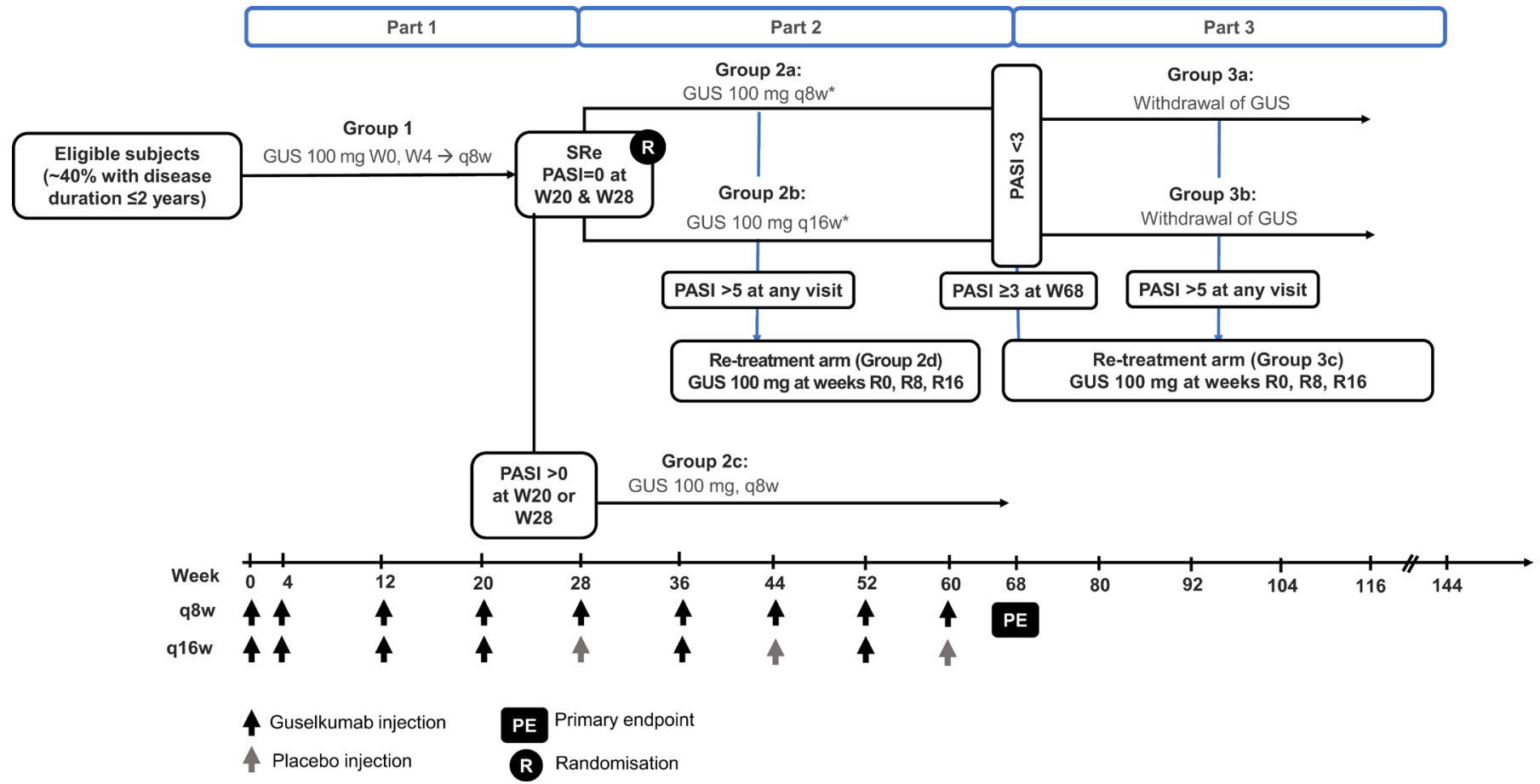

Figure 2 Study setup and design. SRe are defined as participants who receive on-label GUS treatment until W20 and respond with a score of PASI $=0$ at W20 and W28. ${ }^{*}$ Blinded treatment. Group 1: All participants who are enrolled and scheduled to receive GUS $100 \mathrm{mg}$ at W0, W4, then q8w until W28 (study part 1). Group 2a: SRe (PASI score=0 at W20 and W28) randomised to GUS $100 \mathrm{mg}$ q8w at W28-W60 (study part 2). Group 2b: SRe randomised to GUS $100 \mathrm{mg} \mathrm{q16w}$ at W28-W60 (study part 2). Group 2c: non-SRe with a PASI score >0 at W20 and/or W28 who will receive GUS $100 \mathrm{mg}$ q8w at W28-W60 (study part 2). Group 2d: SRe with loss of disease control between W28 and W68, who will enter the retreatment arm and receive GUS $100 \mathrm{mg}$ at R0, R8 and R16 calculated from the date of loss of disease control (study part 2). Group 3a: SRe randomised to GUS $100 \mathrm{mg}$ q8w in study part 2 with withdrawal of GUS at W68 (study part 3). Group 3b: SRe randomised to GUS $100 \mathrm{mg}$ q16w in study part 2 with withdrawal of GUS at W68 (study part 3). Group 3c: SRe with fluctuating disease (PASI score 3-5) at W68 or loss of disease control (PASI score $>5$ ) at any other visit after W68, who will enter the retreatment arm and receive GUS $100 \mathrm{mg}$ at R0, R8 and R16 calculated from the date of loss of disease control (study part 3). GUS, guselkumab; PASI, Psoriasis Area and Severity Index score; q8w, every 8 weeks; q16w, every 16 weeks; R, retreatment week; SRe, super-responder; W, week.

both groups. Participants with a PASI score $>0$ at weeks 20 and/or 28 continue to receive guselkumab $100 \mathrm{mg} \mathrm{q} 8 \mathrm{w}$ until week 60 (defined as study group 2c). Participants in groups $2 \mathrm{a}$ and $2 \mathrm{~b}$ with a PASI score $<3$ at week 68 will enter part 3 of the study and be withdrawn from study medication and followed through week 116. Participants with more substantial loss of response, defined as having PASI score $>5$ at any visit during part 2 or 3 of the study, enter the retreatment arms (groups $2 \mathrm{~d}$ or $3 \mathrm{c}$ ) and receive guselkumab $100 \mathrm{mg}$ at that visit, and then 8 and 16 weeks later. Participants with fluctuating disease (PASI score ranging between 3 and 5) at the eek 68 visit also have the opportunity to enter the retreatment arm (group 3c).

\section{Hypothesis and study evaluations}

Guselkumab treatment may have greater modifying effects on immunopathological mechanisms of disease in participants who are SRe than in those who do not achieve SRe criteria (non-SRe). The hypothesis of this study is that among SRe participants, guselkumab dosed $\mathrm{q} 16 \mathrm{w}$ is non-inferior to guselkumab dosed $\mathrm{q} 8 \mathrm{w}$ as assessed by the proportion of participants with an absolute PASI score $<3$ at week 68 (box 1). The study is also designed to investigate whether dosing guselkumab at these different intervals in SRe may affect maintenance of drug-free control of disease from week 68 through week 116.

Further, the study will investigate whether participants with short disease duration ( $\leq 2$ years) show faster and higher guselkumab responses compared with participants with longer disease duration, and whether participants with shorter disease duration are able to maintain longer drug-free control of disease after guselkumab is withdrawn in study part 3 (box 1).

\section{Statistical analysis}

Statistical analyses in this study will be performed separately for each part of the study and will focus on the comparison of the two randomised treatment groups (ie, group 2a: SRe receiving guselkumab $100 \mathrm{mg}$ q8w vs group 2b: SRe receiving guselkumab $100 \mathrm{mg}$ q16w) in study part 2 . The analyses will be confirmatory for the primary endpoint, and exploratory for the major and all other secondary endpoints. The study is designed to demonstrate that guselkumab $100 \mathrm{mg}$ q16w treatment is non-inferior to guselkumab $100 \mathrm{mg} \mathrm{q} 8 \mathrm{w}$ treatment in SRe, as assessed by the proportion of participants with an absolute PASI score $<3$ at week 68 . A non-inferiority margin of $10 \%$ was chosen based on a minimally clinically 
Box 1 Study objectives

\section{Primary objective}

$\Rightarrow$ To demonstrate that 'super-responders' (SRe; defined as psoriasis patients who receive on-label guselkumab treatment until week 20 and respond with a Psoriasis Area and Severity Index (PASI) score $=0$ at both week 20 and week 28) maintain control of disease until week 68 with a prolonged guselkumab dosing interval of 16 weeks (100 mg q16w). To be demonstrated in study part 2 (see figure 2 for study design).

\section{Secondary objectives \\ $\Rightarrow$ To determine \\ $\Rightarrow$ Whether participants with short disease duration ( $\leq 2$ years) show more rapid and more pronounced guselkumab responses com- pared with participants with longer disease duration, and wheth- er participants with shorter disease duration are able to maintain longer drug-free control of disease after guselkumab withdrawal. To be evaluated in study parts 1,2 and 3 . \\ $\Rightarrow$ Whether guselkumab dosing at different treatment intervals (q8w vs q16w) in SRe from week 28 to week 60 may affect maintenance of drug-free control of disease after 68 weeks of guselkumab treatment. To be evaluated in study part 3 . \\ $\Rightarrow$ The safety and tolerability of guselkumab in participants with moderate-to-severe plaque-type psoriasis.}

meaningful difference. This margin was also used in CNTO1959PSO3009 (the ECLIPSE study) and is therefore considered a valid approach for evaluating noninferiority of a new treatment. ${ }^{64}$

Based on data from CNTO1959PSO3002, the proportion of subjects with an absolute PASI score $<3$ at week 68 is assumed to be $90 \%$ for guselkumab $100 \mathrm{mg} \mathrm{q} 8 \mathrm{w}^{58}$ The expected difference in proportions between the treatment groups (guselkumab $100 \mathrm{mg} \mathrm{q16w}$ minus guselkumab $100 \mathrm{mg} \mathrm{q} 8 \mathrm{w}$ ) is $0 \%$. When the sample size in each group is 112, a two-group, large-sample normal approximation Wald Z-test of proportions with a one-sided 0.05 significance level will have $80 \%$ power to reject the null hypothesis that the guselkumab $100 \mathrm{mg}$ q16w treatment is inferior to the guselkumab $100 \mathrm{mg} \mathrm{q} 8 \mathrm{w}$ treatment. The confirmatory significance level for non-inferiority testing is fixed at a one-sided $\alpha=0.05$.

Descriptive statistics will include counts and proportions for categorical data, mean, SD, median, IQR and range for continuous data. The two-group, large-sample normal approximation Wald Z-test with Mantel-Haenszel stratum weights for 'disease duration' will be used to compare the proportion of participants responding to treatment. Continuous response parameters will be compared using an analysis of variance model with fixed effects for treatment group, disease duration and baseline (week 0) value as a covariate. Time-to-event endpoints will be analysed using Kaplan-Meier product limit methods to estimate survival distributions and median time-to-event.

\section{Mechanistic biomarker substudies}

Four substudies are conducted to further evaluate the pharmacological effects of guselkumab. They evaluate
Box 2 Objectives of mechanistic biomarker substudies

\section{Exploratory objectives}

$\Rightarrow$ To characterise:

$\Rightarrow$ Immune cellular features at baseline (week 0) and changes (quantitative and qualitative characterisation) in lesional skin of participants during treatment with guselkumab as determined by fluorescence activated cell sorting (FACS)-based analysis. To be explored in substudy 1.

$\Rightarrow$ Immune cellular features in the blood of participants at baseline (week 0), and changes (quantitative and qualitative characterisation) during and after treatment with guselkumab as determined by FACS-based analysis. To be explored in substudy 1 .

$\Rightarrow$ Molecular (gene expression) changes in the skin of participants treated with guselkumab as determined by RNA sequencing (RNAseq) and quantitative PCR. To be explored in substudy 2.

$\Rightarrow$ Tissue immunopathological changes in the skin of participants during and after treatment with guselkumab as determined by immunohistochemistry/immunofluorescence/in situ hybridisation. To be explored in substudy 2 .

$\Rightarrow$ Effects of guselkumab treatment on serum biomarkers as determined by immunoassays. To be explored in substudy 3 .

$\Rightarrow$ The association between changes in the various exploratory biomarker endpoints and (1) efficacy of guselkumab, (2) duration of psoriasis, (3) maintenance of response after stopping guselkumab treatment and (4) ability to achieve a PASI 100 response at weeks 20 and 28 (super responder status). To be explored in all substudies.

various pharmacological/clinical response relationships allowing assessment of interindividual variability in clinical outcomes and possible identification of subject population groups that may show particularly differentiating responses to guselkumab. The substudies also aim to further understand the mechanism of action (MoA) of guselkumab at the molecular and cellular levels during and after treatment. Changes in gene expression as well as quantitative and qualitative changes in different types of immune cells are evaluated in skin biopsies and blood (box 2).

In substudy 1 (cellular MoA substudy), $6 \mathrm{~mm}$ skin biopsy samples are collected from non-lesional skin at baseline (week 0) and lesional skin at weeks 0, 4, 28 and 68 from approximately 60 participants. In addition, if participants lose control of disease (having a PASI $>5$ ) in study parts 2 or 3 and go into retreatment, an additional biopsy is taken from an active lesional plaque. Biopsies will be dissociated into single-cell suspension and subjected to fluorescenceactivated cell sorting (FACS)-based immunophenotyping analysis of $\mathrm{T}$ cells, macrophages and dendritic cells. Further, whole blood samples for the isolation of peripheral blood mononuclear cells are collected from consenting participants for subsequent immunophenotyping analyses by FACS. In substudy 2 (gene expression substudy), $6 \mathrm{~mm}$ skin biopsies are collected in a subset of participants (target $\mathrm{n}=100$ ) at selected sites to evaluate gene expression profiles and cellular content by surface protein staining. Immune staining techniques include H\&E stain, immunohistochemistry and 
immunofluorescence analyses. For substudy 3 (serum analysis), serum samples are collected from all participants at weeks $0,4,28,68$ and 80 to assess pharmacodynamic effects on blood protein analytes associated with the response to guselkumab as well as markers related to psoriasis. Measurements include, but are not limited to, serum IL-17A, IL-17F, IL-22 and beta defensin-2 (BD-2) levels. Genetic variation can be an important contributing factor to interindividual differences in drug distribution and response and can also serve as a marker for disease susceptibility and prognosis. Substudy 4 (genetic analyses) may help to identify subpopulations that respond differently to a drug. The goal of the genetic (DNA) analysis is to identify genetic factors that may influence responses to guselkumab in the populations of psoriasis patients studied.

Together, these substudies will relate immunopathological mechanisms to clinical course and characteristics of plaque psoriasis and may thus provide insights into individualised guselkumab treatment algorithms. This could include identification of predictors of optimal responses to guselkumab.

Together with the underlying mechanistic biomarker substudies, GUIDE evaluates whether selective IL-23 blockade exerts significant modifying effects on disease pathophysiology by studying and correlating clinical (eg, duration of disease), serological (eg, drug serum concentration) and immunopathophysiological (eg, balance of $\mathrm{T}_{\text {reg }}$ and $\mathrm{T}_{\mathrm{RM}}$ ) disease parameters in patients treated with guselkumab for a year followed by withdrawal of treatment, and evaluates whether these effects are more pronounced in patients with shorter disease duration.

\section{ETHICS AND DISSEMINATION}

The GUIDE study was designed and will be conducted and reported in accordance with the International Conference on Harmonisation Harmonised Tripartite Guidelines for Good Clinical Practice, with applicable local regulations, and in accordance with the Declaration of Helsinki. A positive opinion has been obtained from the ethics committee Medical Council Hamburg (PVN5925).

All patients are required to provide written informed consent. A separate consent form will be signed by patients taking part in the optional mechanistic substudies 1 and 2 and the optional pharmacogenetic testing (substudy 4).

All study results will be published. Data for the primary endpoint analysis at week 68 are expected to be available in 2022; final results are expected to be published in 2024.

\section{Status and perspective}

GUIDE started enrolment in February 2019 in Germany and in December 2019 in France. Full enrolment was achieved in November 2020. Final database lock is expected in 2023.

\section{Patient and public involvement}

Patients or the public will not be involved in the design, or conduct, or reporting, or dissemination plans of this research.

\section{Author affiliations}

${ }^{1}$ Department of Medicine, Division of Dermatology and Venereology, Karolinska Institute, Stockholm, Sweden

${ }^{2}$ Dermatologikum Hamburg GmbH, Hamburg, Germany

${ }^{3}$ University Hospital Frankfurt am Main, Frankfurt, Germany

${ }^{4}$ Department of Dermatology, and Interdisciplinary Center for Chronic Inflammatory

Diseases, University Hospital Heidelberg, Heidelberg, Germany

${ }^{5}$ Dermatological Practice, Potsdam, Germany

${ }^{6}$ Janssen-Cilag GmbH, Neuss, Germany

${ }^{7}$ Janssen Research \& Development, LLC, San Diego, California, USA

${ }^{8}$ Janssen-Cilag, Vienna, Austria

${ }^{9}$ TautScience and Service Ltd, Konstanz, Germany

${ }^{10}$ Institute for Health Services Research in Dermatology and Nursing, University Medical Center Hamburg-Eppendorf, Hamburg, Germany

Acknowledgements The authors wish to thank Sonja Kock (BioMedical Communications, Giekau, Germany) and Anne-Kathrin Wegner (Medical Communication, Neuss, Germany) for medical writing and support in the preparation of this manuscript, Steven Fakharzadeh (Janssen Global, Horsham, Pennsylvania, USA) and Betty Yang (Janssen Global, Horsham, Pennsylvania, USA) for critical review of the manuscript, the GUIDE study team, the investigators and participating patients.

Contributors KE, PW, AP, KA, SW, EJM-E, HB, FJHT and KR conceived the study. $A P, K A, S W, H B, F J H T$ and $K R$ contributed to the development of the study design of methodology; KE, PW, AP, KS, KA, SW, EJM-E, FJHT and KR conducted the research; PW, KS, SW, EJM-E and HB directed and coordinated research activity; AP, KS, SW and EJM-E contributed to formal data analysis/validation; SW, EJM-E, FJHT and KR contributed to writing the manuscript. All authors reviewed and approved the final version of the paper.

Funding The GUIDE study is funded by Janssen-Cilag, Germany and Janssen, France. Editorial support was provided by Cello Health MedErgy and funded by Janssen-Cilag, Germany and Janssen, France.

Competing interests $\mathrm{KE}$ reports grants and personal fees from Janssen during the conduct of the study, grants from AbbVie, LEO Pharma, UCB and Novartis, and personal fees from AbbVie, Almirall, BMS, LEO Pharma, Lilly, Sanofi, UCB, Galderma and Novartis outside the submitted work; PW reports receiving honoraria as consultant or speaker from the following companies involved in the development or distribution of drugs for psoriasis: AbbVie, Almirall, Biogen, Celgene, Eli Lilly, Janssen, LEO Pharma, Medac and Novartis, and honoraria received by his institution for active participation in clinical studies sponsored by Janssen, AbbVie and Eli Lilly; AP has no conflicts of interest to report; KS reports conducting clinical studies during the past 36 months with the following companies: AbbVie, Almirall, Boehringer, Celgene, Chugai, Galapagos, Galderma, Janssen-Cilag, LEO Pharma, Lilly, Merck Sharp \& Dohme Corp., Novartis Regeneron and UCB Pharma; KA reports honoraria for participation in advisory boards, consultation, clinical trials or as speaker from AbbVie, Almirall, Antabio, Bayer, BMS, Euroimmune, Emphasis, Emeritipharma, Galderma, Janssen, La Roche-Posay, LEO Pharma, L'Oréal, Novartis, Parexel International, Pierre Fabre, Roxall, RG, Sanofi Genzyme, TFS Trial Form Support and UCB; SW is a full-time employee of Janssen-Cilag Germany; EJM-E is a full-time employee of Johnson \& Johnson, and is listed as an inventor on a patent application related to uses of guselkumab to treat psoriasis, pending; HB reports personal fees from Janssen-Cilag Germany during the conduct of the study, and personal fees from Janssen-Cilag Germany outside the submitted work; FJHT reports personal fees from Janssen-Cilag, Germany during the conduct of the study and personal fees from Janssen-Cilag, Germany outside the submitted work; KR reports grants and personal fees from Janssen during the conduct of the study, grants from AbbVie, Lilly, LEO Pharma, UCB, Pfizer, Affibody, Biogen-Idec, Boehringer Ingelheim Pharma, BMS, Celgene, Covagen, Forward Pharma, Fresenius Medical Care, Galapagos, Kyowa Kirin, Medac, Merck Sharp \& Dohme, Milteny, Novartis, Ocean Pharma, Sandoz, Sanofi, Sun Pharma, Takeda and XBiotech; personal fees from AbbVie, Lilly, LEO Pharma, UCB, Pfizer, Amgen, Affibody, Biogen-Idec, Boehringer Ingelheim Pharma, BMS, Celgene, Covagen, Forward Pharma, GSK, Kyowa Kirin, Medac, Merck Sharp \& Dohme Corp, Novartis, Ocean Pharma, Samsung Bioepis, Sandoz, Sanofi, Takeda, Valeant and Xenoport outside the submitted work. 
Patient and public involvement Patients and/or the public were not involved in the design, or conduct, or reporting, or dissemination plans of this research.

Patient consent for publication Not required.

Provenance and peer review Not commissioned; externally peer reviewed.

Data availability statement Requests for access to the study data can be submitted through Yale Open Data Access (YODA) Project site at yoda.yale.edu at: https://www.janssen.com/clinical-trials/transparency.

Open access This is an open access article distributed in accordance with the Creative Commons Attribution Non Commercial (CC BY-NC 4.0) license, which permits others to distribute, remix, adapt, build upon this work non-commercially, and license their derivative works on different terms, provided the original work is properly cited, appropriate credit is given, any changes made indicated, and the use is non-commercial. See: http://creativecommons.org/licenses/by-nc/4.0/.

\section{ORCID iD}

Kristian Reich http://orcid.org/0000-0001-5248-4332

\section{REFERENCES}

1 Takeshita J, Grewal S, Langan SM, et al. Psoriasis and comorbid diseases: epidemiology. J Am Acad Dermatol 2017;76:377-90.

2 Hawkes JE, Yan BY, Chan TC, et al. Discovery of the IL-23/IL17 signaling pathway and the treatment of psoriasis. J Immunol 2018;201:1605-13.

$3 \mathrm{Kim}$ J, Krueger JG. Highly effective new treatments for psoriasis target the IL-23/type 17 T cell autoimmune axis. Annu Rev Med 2017;68:255-69.

4 World Health Organization. Global report on psoriasis, 2016. Available: https://apps.who.int/iris/handle/10665/204417

5 Mrowietz U, de Jong EMGJ, Kragballe K, et al. A consensus report on appropriate treatment optimization and transitioning in the management of moderate-to-severe plaque psoriasis. J Eur Acad Dermatol Venereol 2014;28:438-53.

6 Blome C, Gosau R, Radtke MA, et al. Patient-relevant treatment goals in psoriasis. Arch Dermatol Res 2016;308:69-78.

7 Mrowietz U, Kragballe K, Reich K, et al. Definition of treatment goals for moderate to severe psoriasis: a European consensus. Arch Dermatol Res 2011;303:1-10.

8 Chan TC, Hawkes JE, Krueger JG. Interleukin 23 in the skin: role in psoriasis pathogenesis and selective interleukin 23 blockade as treatment. Ther Adv Chronic Dis 2018:9:111-9.

9 Schäkel K, Schön MP, Ghoreschi K. Pathogenese der Psoriasis vulgaris [Pathogenesis of psoriasis vulgaris]. Der Hautarzt 2016;67:422-31.

10 Chiricozzi A, Romanelli P, Volpe E, et al. Scanning the immunopathogenesis of psoriasis. Int J Mol Sci 2018;19:179.

11 Girolomoni G, Strohal R, Puig L, et al. The role of IL-23 and the IL-23/ TH17 immune axis in the pathogenesis and treatment of psoriasis. $J$ Eur Acad Dermatology Venereol 2017;31:1616-26.

12 Bovenschen HJ, van de Kerkhof PC, van Erp PE, et al. Foxp3+ regulatory $\mathrm{T}$ cells of psoriasis patients easily differentiate into IL17A-producing cells and are found in lesional skin. J Invest Dermatol 2011;131:1853-60.

13 Kannan AK, Su Z, Gauvin DM, et al. IL-23 induces regulatory T cell plasticity with implications for inflammatory skin diseases. Sci Rep 2019;9:17675.

14 Soler DC, McCormick TS. The dark side of regulatory T cells in psoriasis. J Invest Dermatol 2011;131:1785-6.

15 Yang L, Li B, Dang E, et al. Impaired function of regulatory T cells in patients with psoriasis is mediated by phosphorylation of STAT3. $J$ Dermatol Sci 2016;81:85-92.

16 Cheuk S, Wikén M, Blomqvist L, et al. Epidermal Th22 and Tc17 cells form a localized disease memory in clinically healed psoriasis. $J$ Immunol 2014;192:3111-20.

17 Clark RA. Resident memory T cells in human health and disease. Sci Transl Med 2015;7:269rv1.

18 Reich K, Papp KA, Armstrong AW, et al. Safety of guselkumab in patients with moderate-to-severe psoriasis treated through 100 weeks: a pooled analysis from the randomized VOYAGE 1 and VOYAGE 2 studies. Br J Dermatol 2019;180:1039-49.

19 Reich K, Griffiths CEM, Gordon KB, et al. Maintenance of clinical response and consistent safety profile with up to 3 years of continuous treatment with guselkumab: Results from the VOYAGE 1 and VOYAGE 2 trials. J Am Acad Dermatol 2020;82:936-45.

20 Ghilardi N, Kljavin N, Chen Q, et al. Compromised humoral and delayed-type hypersensitivity responses in IL-23-deficient mice. $J$ Immunol 2004;172:2827-33.
21 Gooderham MJ, Papp KA, Lynde CW. Shifting the focus - the primary role of IL-23 in psoriasis and other inflammatory disorders. $J$ Eur Acad Dermatology Venereol 2018;32:1111-9.

22 Di Meglio P, Nestle FO. The role of IL-23 in the immunopathogenesis of psoriasis. F1000 Biol Rep 2010;2:40.

23 Duvallet E, Semerano L, Assier E, et al. Interleukin-23: a key cytokine in inflammatory diseases. Ann Med 2011;43:503-11.

24 Puig L. The role of IL 23 in the treatment of psoriasis. Expert Rev Clin Immunol 2017;13:525-34.

25 Sun R, Hedl M, Abraham C. IL23 induces IL23R recycling and amplifies innate receptor-induced signalling and cytokines in human macrophages, and the IBD-protective IL23R R381Q variant modulates these outcomes. Gut 2020;69:264-73.

26 Longman RS, Diehl GE, Victorio DA, et al. $\mathrm{CX}_{3} \mathrm{CR} 1^{+}$mononuclear phagocytes support colitis-associated innate lymphoid cell production of IL-22. J Exp Med 2014;211:1571-83.

27 Gaffen SL, Jain R, Garg AV, et al. The IL-23-IL-17 immune axis: from mechanisms to therapeutic testing. Nat Rev Immunol 2014;14:585-600.

28 Durant L, Watford WT, Ramos HL, et al. Diverse targets of the transcription factor STAT3 contribute to T cell pathogenicity and homeostasis. Immunity 2010;32:605-15.

29 Ivanov II, McKenzie BS, Zhou L, et al. The orphan nuclear receptor RORgammat directs the differentiation program of proinflammatory IL-17+ T helper cells. Cell 2006;126:1121-33.

30 Cheuk S, Schlums H, Gallais Sérézal I, et al. CD49a expression defines tissue-resident $C D 8^{+} \mathrm{T}$ cells poised for cytotoxic function in human skin. Immunity 2017;46:287-300.

31 Park CO, Kupper TS. The emerging role of resident memory T cells in protective immunity and inflammatory disease. Nat Med 2015;21:688-97.

32 Clark RA. Gone but not forgotten: lesional memory in psoriatic skin. J Invest Dermatol 2011;131:283-5.

33 Clark RA. Skin-resident T cells: the ups and downs of on site immunity. J Invest Dermatol 2010;130:362-70.

$34 \mathrm{Wu} \mathrm{H}$, Liao W, Li Q, et al. Pathogenic role of tissue-resident memory T cells in autoimmune diseases. Autoimmun Rev 2018;17:906-11.

35 Bhushan M, Bleiker TO, Ballsdon AE, et al. Anti-E-selectin is ineffective in the treatment of psoriasis: a randomized trial. $\mathrm{Br} J$ Dermatol 2002;146:824-31

36 Boyman $\mathrm{O}$, Conrad $\mathrm{C}$, Tonel $\mathrm{G}$, et al. The pathogenic role of tissueresident immune cells in psoriasis. Trends Immunol 2007;28:51-7.

37 Boyman O, Hefti HP, Conrad C, et al. Spontaneous development of psoriasis in a new animal model shows an essential role for resident T cells and tumor necrosis factor-alpha. J Exp Med 2004;199:731-6.

38 Suárez-Fariñas M, Fuentes-Duculan J, Lowes MA, et al. Resolved psoriasis lesions retain expression of a subset of disease-related genes. J Invest Dermatol 2011;131:391-400.

39 Gallais Sérézal I, Hoffer E, lgnatov B, et al. A skewed pool of resident $T$ cells triggers psoriasis-associated tissue responses in neverlesional skin from patients with psoriasis. J Allergy Clin Immunol 2019;143:1444-54.

40 Kurihara K, Fujiyama T, Phadungsaksawasdi P, et al. Significance of IL-17A-producing $\mathrm{CD}^{+} \mathrm{CD} 103^{+}$skin resident memory $\mathrm{T}$ cells in psoriasis lesion and their possible relationship to clinical course. $J$ Dermatol Sci 2019;95:21-7.

41 Matos TR, O'Malley JT, Lowry EL, et al. Clinically resolved psoriatic lesions contain psoriasis-specific IL-17-producing $\alpha \beta$ T cell clones. J Clin Invest 2017;127:4031-41.

42 Casciano F, Pigatto PD, Secchiero P, et al. T cell hierarchy in the pathogenesis of psoriasis and associated cardiovascular comorbidities. Front Immunol 2018:9:1390.

43 Mehta H, Mashiko S, Angsana J. Differential changes in inflammatory mononuclear phagocyte and T cell profiles within psoriatic skin during treatment with Guselkumab versus Secukinumab. J Invest Dermatol 2021;141:1707-18.

44 Ali N, Rosenblum MD. Regulatory T cells in skin. Immunology 2017;152:372-81.

45 Lee GR. The balance of Th17 versus Treg cells in autoimmunity. Int $J$ Mol Sci 2018;19:730.

46 Zhou X, Bailey-Bucktrout SL, Jeker LT, et al. Instability of the transcription factor FOXP3 leads to the generation of pathogenic memory T cells in vivo. Nat Immunol 2009;10:1000-7.

47 Chen X, Oppenheim JJ. Th17 cells and Tregs: unlikely allies. J Leukoc Biol 2014;95:723-31.

48 Van Herck MA, Weyler J, Kwanten WJ, et al. The differential roles of $\mathrm{T}$ cells in non-alcoholic fatty liver disease and obesity. Front Immunol 2019;10:82.

49 Sanchez Rodriguez R, Pauli ML, Neuhaus IM, et al. Memory regulatory $T$ cells reside in human skin. J Clin Invest 2014:124:1027-36. 
50 Sugiyama H, Gyulai R, Toichi E, et al. Dysfunctional blood and target tissue CD4+CD25high regulatory $T$ cells in psoriasis: mechanism underlying unrestrained pathogenic effector $T$ cell proliferation. $J$ Immunol 2005:174:164-73.

51 Aletaha D, Smolen JS. The definition and measurement of disease modification in inflammatory rheumatic diseases. Rheum Dis Clin North Am 2006;32:9-44.

52 Danese S, Fiorino G, Peyrin-Biroulet L. Early intervention in Crohn's disease: towards disease modification trials. Gut 2017;66:2179-87.

53 Girolomoni G, Griffiths CEM, Krueger J, et al. Early intervention in psoriasis and immune-mediated inflammatory diseases: a hypothesis paper. J Dermatolog Treat 2015;26:103-12.

54 Nast A, Boehncke W-H, Mrowietz U. S3-Leitlinie zur Therapie der Psoriasis vulgaris Update 2011 [S3-guidelines for the treatment of psoriasis vulgaris update 2011]. J Dtsch Dermatol Ges 2011;9:S1-104.

55 van den Reek JMPA, Seyger MMB, van Lümig PPM, et al. The journey of adult psoriasis patients towards biologics: past and present - Results from the BioCAPTURE registry. J Eur Acad Dermatol Venereol 2018;32:615-23.

56 Blauvelt A, Papp KA, Griffiths CEM, et al. Efficacy and safety of guselkumab, an anti-interleukin-23 monoclonal antibody, compared with adalimumab for the continuous treatment of patients with moderate to severe psoriasis: results from the phase III, doubleblinded, placebo- and active comparator-controlled voyage 1 trial. $J$ Am Acad Dermatol 2017;76:405-17.

57 Papp KA, Blauvelt A, Kimball AB, et al. Patient-reported symptoms and signs of moderate-to-severe psoriasis treated with guselkumab or adalimumab: results from the randomized voyage 1 trial. J Eur Acad Dermatol Venereol 2018;32:1515-22.

58 Reich K, Armstrong AW, Foley P, et al. Efficacy and safety of guselkumab, an anti-interleukin-23 monoclonal antibody, compared with adalimumab for the treatment of patients with moderate to severe psoriasis with randomized withdrawal and retreatment: results from the phase III, double-blind, placebo- and active comparatorcontrolled voyage 2 trial. J Am Acad Dermatol 2017;76:418-31.

59 Griffiths CEM, Papp KA, Song M, et al. Maintenance of response through 5 years of continuous guselkumab treatment: results from the phase 3 VOYAGE 1 trial. Poster presented at the 16th annual coastal dermatology symposium, October 15-16 2020.

60 Gordon K, Armstrong A, Foley Y. Long-term efficacy of guselkumab treatment after drug withdrawal and retreatment in patients with moderate to severe plaque psoriasis: results from VOYAGE 2. presented at AAD annual meeting, 16-18 February Abstract 6748 2018.

61 Griffiths CE, Papp K, Kimball AB. Two-year efficacy and safety of guselkumab for treatment of moderate-to-severe psoriasis: phase 3 VOYAGE 1 trial (Abstract AB0912). Ann Rheum Dis 2018;77:1580.

62 Gordon KB, Armstrong AW, Foley P, et al. Guselkumab efficacy after withdrawal is associated with suppression of serum IL-23-regulated IL-17 and IL-22 in psoriasis: VOYAGE 2 study. J Invest Dermatol 2019;139:2437-46.

63 Liu X, Branigan P, Chen Y, et al. Identification of clinical and biomarker parameters associated with long-term maintenance of PASI 90 response following guselkumab treatment-withdrawal in psoriasis. Poster presented at the 27th EADV Congress, 12-16 September 2018, Paris, France Abstract:P1894 2018.

64 Reich K, Armstrong AW, Langley RG, et al. Guselkumab versus secukinumab for the treatment of moderate-to-severe psoriasis (eclipse): results from a phase 3 , randomised controlled trial. Lancet 2019;394:831-9.

65 Ghoreschi K, Laurence A, Yang XP, et al. T helper 17 cell heterogeneity and pathogenicity in autoimmune disease. Trends Immunol 2011;32:395-401. 